\title{
MUCIA TERCIA: \\ MATRONA ROMANA, MEDIADORA POLÍTICA. UN ESTADO DE LA CUESTIÓN
}

\author{
Gustavo A. Vivas García* \\ Universidad de La Laguna \\ gusgarvi@gmail.com \\ orcid.org/0000-0002-2820-7199
}

\author{
A Isabel García Gálvez (†) \\ A quién si no, después de tantos años...
}

\section{RESUMEN}

En su reseña de La Revolución Romana, Arnaldo Momigliano menciona a Mucia Tercia, matrona romana del siglo I, conocida por ser esposa de Pompeyo (106-48) y madre de Sexto (ca. 66-35). Mucia fue un personaje de una fascinante existencia vital y una auténtica prueba viviente de la importancia de la mujer como agente político de influencia y repercusión en el último siglo de la República romana, que vio otros ejemplos de mujeres de similar trascendencia en la esfera de la alta política: Servilia, Fulvia; Julia, hija del futuro princeps, o la misma hermana de éste, Octavia, entre otras.

Palabras ClaVe: Mucia Tercia, matrona romana, República tardía, historia de las mujeres.

MUCIA TERCIA:

ROMAN MATRON, POLITICAL MEDIATOR.

A STATE OF THE QUESTION

\section{ABSTRACT}

In his review of The Roman Revolution $[R R]$, Arnaldo Momigliano alludes to the Roman matron Mucia Tercia, matron of the $1^{\text {st }}$ century, known for being Pompey's spouse (106-48) and mother of Sextus (ca.66-35). Mucia was a figure with fascinating life experience and a real proof of the importance of women as political agents of influence and impact in the last century of the Roman Republic. Mucia witnessed other examples of women with significant importance in the high political sphere of the Republic: Servilia, Fulvia; Julia, daughter of the future princeps; or the very same princeps'sister Octavia, among others.

KeY WORDS: Mucia Tercia, Roman matron, Late Republic, Women's history.

\section{INTRODUCCIÓN}

En su conocida reseña sobre The Roman Revolution $[R R]$, una de las primeras opiniones puestas por escrito sobre este libro clásico que todavía hoy se lee con 
frescura e interés, Arnaldo Momigliano ${ }^{1}$ se refiere a una matrona romana quizá desconocida para muchos de sus lectores. Nos referimos a Mucia Tercia, matrona romana del siglo I, conocida por ser esposa de Pompeyo (106-48) y la madre de su hijo Sexto (ca. 66-35). Mucia fue un personaje de una fascinante existencia vital y una auténtica prueba viviente de la importancia de la mujer como agente político de influencia y repercusión en ese último siglo de la República romana, que vio otros ejemplos de mujeres de similar trascendencia en la esfera de la alta política: Servilia, Fulvia; Julia, hija del futuro princeps, o la misma hermana de éste, Octavia entre otras ${ }^{2}$.

La referencia del insigne polígrafo italiano es escueta, una simple línea: "The attempt of Mucia Tercia to mediate between Sex. Pompeius and Octavian is not recorded [en $R R$ ]" ". Momigliano está enumerando en ese punto de la reseña una serie de hechos que figuran en las fuentes pero que Syme no menciona en su célebre monografía y éste es uno de ellos.

Se refiere, en concreto, a las labores de mediación política llevadas a cabo por Mucia hasta en dos ocasiones para que su hijo Sexto y Octaviano alcanzaran algún tipo de acuerdo. En primer lugar en 40, lo que motivó un enfriamiento de la tensión desatada entre ambos y el casamiento de Octaviano con Escribonia, hermana del suegro de Sexto Pompeyo (D.C. 48,16,2-3), y un año después en la primavera de 39, cuando unos alborotadores en la capital amenazaron con quemar a Mucia en su propia casa y Octavio le pidió, de nuevo, a esta matrona que acudiera como negociadora de paz ante su hijo en nombre suyo (App. BC 5,69). Estas gestiones dieron su fruto en un tratado acordado poco tiempo después en un episodio muy bien documentado en las fuentes literarias, en Apiano y Plutarco especialmente. Sobre ambos episodios volveremos más adelante con detenimiento en este trabajo.

Tras haber leído decenas de veces el texto de Momigliano, mi objetivo es intentar averiguar algo más sobre quién fue esta mujer cuya existencia es tan desconocida como atractiva. Desde el principio intuí que poseía una importancia asimilable a las mencionadas más arriba en cuanto a significación e influencia en el ámbito de la historia política. Creo que los complejos hechos acaecidos durante estos años

* Universidad de La Laguna. UDI de Prehistoria, Arqueología e Historia Antigua. Facultad de Humanidades. 38200 Tenerife - Islas Canarias. A menos que se indique lo contrario, todas las fechas de este trabajo son AEC. Agradezco al Prof. Anthony R. Birley y al Prof. José A. Delgado Delgado la atenta lectura de este trabajo y sus sugerencias. Y, por supuesto, mi gratitud al Prof. Dr. José A. González Marrero.

${ }^{1}$ Momigliano, 1940: 75-80. Cita en 76.

${ }^{2}$ Vd. por ejemplo Fischer, 1999; Brennan, 2012; Vivas García, 2013 o Harders, 2015, para una buena perspectiva de conjunto. Para obtener una visión aguda e incisiva de la nobilitas tardorepublicana véase a Hölkeskamp, 2004 y 2017; entre otros muchos trabajos suyos, y también a Zmeskal, 2009.

${ }^{3} \mathrm{Vd}$. n.1. 
tumultuosos de la historia de la antigua Roma pueden comprenderse mejor si tratamos de poner al día lo que las fuentes y la investigación actual nos cuentan de figuras tan apasionantes como la de Mucia Tercia. Este es el propósito de las líneas que siguen.

\section{UN ESTADO DE LA CUESTIÓN}

Mucia Tercia nació en una familia de la más linajuda aristocracia romana aproximadamente en los años finales del s. II o, más probablemente, en los iniciales del siglo I (Stegmann, 2000: 423-424). Ninguna fuente nos proporciona la fecha exacta de su nacimiento. Su padre fue Q. Mucio Escévola (cos. 95, MRR II, 11). Su cognomen no prueba que tuviera dos hermanas mayores (cf. Fluss, 1933: 449), pero no es descartable aunque tenga relativa o escasa importancia. Quizá el cognomen lo usara para diferenciarse de sus tías pero ninguna fuente nos aclara este particular.

Q. Cecilio Metelo Céler (cos. 60, MRR II, 182) y Q. Cecilio Metelo Nepote (cos. 57, MRR II, 199) fueron sus hermanastros tras el casamiento de su madre, Licinia Crasa o Prima, con el padre de ambos Metelos, Q. Cecilio Metelo Nepote (cos. 98, $M R R$ II, 4). Cicerón y Dion Casio apuntan que este casamiento, precedido de un tumultuoso divorcio de Escévola, causó cierto escándalo entre la "buena sociedad" romana de su época (Cic. Ad. Fam. 5,2; D.C. 37,49,3). Ambos Metelos fueron también primos suyos (DPRR, Mucia [28] Tertia).

Mucia ha pasado a la Historia con mayúsculas por ser la tercera de las cinco esposas de Pompeyo el Grande. Además, tal y como señalaremos a lo largo de este trabajo, Mucia fue también una matrona de gran influencia en su época por otras razones. Su papel mediador en diversos episodios de la política contemporánea, destacado por la gran mayoría de las fuentes del período, es uno de los factores más descollantes en su biografía.

Esta influyente mujer contrajo matrimonio con Pompeyo hacia el 80 (Masi Doria, 2014: 496) o, mejor aún en 81 (Seager, 2002: 29 y 185). Haley por su parte (1985: 50) sitúa el casamiento en 79. Tras su victoria sobre Cayo Mario fue Sila, en tanto que dictator el que proporcionó a Pompeyo esta nueva esposa para asegurarse su lealtad, siendo elegida de entre el clan de los Metelos, tras el fallecimiento el mismo año 81 de su segunda esposa Emilia, poco después de dar a luz.

Con esta unión, que poseía una interesante lectura en el plano político, Pompeyo consiguió una serie de conexiones al más alto nivel de decisión entre las familias más influyentes de la República, en concreto con el relevante clan de los Metelli. Esta unión marital de Pompeyo fue singular por su duración, ya que el matrimonio pervivió hasta el 62 y fue el de mayor duración de los cinco que contrajo el general. La unión fue también especial por las circunstancias peculiares de su disolución, que analizaremos en profundidad más adelante. Las fuentes especifican claramente que Mucia proporcionó a Pompeyo una descendencia de tres hijos: una niña, Pompeya, que casaría con Fausto Cornelio Sila (q. 54); y dos varones, Sexto y Cneo (Ascon., Pro M. Scaur. 17; Suet., César 50,1 y D.C. 37,49,3). Las mismas fuentes nos indican 
la fecha del nacimiento de Sexto, el más famoso de los tres4. Según Apiano $(B C 5,144)$, al que sigue Fluss (1933: 449), Sexto debió de nacer hacia 75 y murió en 35 con cuarenta años de edad. Gabba, sin embargo (1970: comm. ad. loc.), sitúa su nacimiento en 66 o 68, con lo que tendría poco más de treinta años cuando falleció.

Después de casi veinte años de matrimonio y tres vástagos en común, la pareja se divorció en 62 . Entre los años 76 y 62 Pompeyo pasó la mayor parte de su tiempo fuera de la Urbs, emprendiendo campañas contra Sertorio en Hispania, en el Mediterráneo contra los piratas o en Oriente luchando frente a Mitrídates VI Eupator, rey del Ponto (Seager, 2002). En 62, a la vuelta a Roma tras el bellum Mithridaticum, Pompeyo que por entonces era ya un veterano consular, se divorció de Mucia en diciembre de 62 alegando como motivo el adulterio. Seager escribe (ibíd.: 76): "His first action [de Pompeyo a la vuelta de Oriente] was to divorce his wife Mucia, on whose conduct during his absence rumour cast the gravest suspicion". Por tanto, uno de los más recientes y mejores biógrafos del general parece tenerlo claro. Recordemos ahora que este episodio rememora en cierta medida el traumático divorcio y posterior casamiento de su madre, acontecido casi cuarenta años antes.

Autores recientes, como Haley (1985: 50), opinan que las fuentes no proporcionan una razón clara para la disolución del matrimonio. Sin embargo Asconio, Plutarco y Suetonio se hacen eco de las supuestas infidelidades cometidas por Mucia. Asconio (Pro M. Scaur. 17) cuenta que hacia 54, M. Emilio Escauro (pr. 56, MRR II, 208) que había contraído matrimonio con Mucia tras el divorcio de Pompeyo, se había ganado la enemistad de éste puesto que se burlaba de él a causa de la infidelidad que había sufrido.

Por su parte Plutarco (Pompeyo, 42) señala de manera incontestable que: "Mucia le había sido infiel durante su ausencia. Mientras estuvo lejos, Pompeyo mostró desprecio por las informaciones sobre esta cuestión; pero cuando estuvo cerca de Italia y, según parece, tuvo más tiempo para reflexionar, envió a su esposa un acta de divorcio, sin comunicarle por escrito, ni entonces ni después, los motivos por los cuales se divorciaba de ella (...)" (BCG, trad. Salvador Bueno Morillo). La cita sigue en Plutarco refiriendo que Cicerón, en sus cartas, explica la causa del divorcio. Lo cierto es la única referencia directa en Cicerón al divorcio de Mucia y Pompeyo se encuentra en una de sus cartas a Ático, fechada escasas semanas después del episodio: el 1 de enero de 61 (ad Att., 1,12,3) donde escribe: "Me consta que Pompeyo es muy amigo mío. Se aprueba calurosamente su divorcio de Mucia" (BCG, trad. Miguel Rodríguez-Pantoja Márquez).

${ }^{4}$ Tras la monografía clásica de Hadas, 1930; un estudio demasiado positivista y de carácter biográfico que añade poco al análisis en clave histórico-política de la figura de Sexto Pompeyo; en los últimos años han aparecido excelentes trabajos que proporcionan una visión más integral y profunda sobre el personaje. Cabe mencionar aquí como compendio de todos ellos el estudio paradigmático de Welch, 2012. 
Suetonio (César, 50) menciona a Julio César como amante de la aristócrata, siendo la única fuente que indica este hecho. Sabemos que el divorcio se convertía en una afrenta para toda la familia, máxime si existían cuestiones de infidelidad por medio, como al parecer ocurrió en este caso. No olvidemos que la élite dirigente romana es una sociedad imbuida de un marcado carácter aristocrático y competitivo, donde el "honor" constituía un elemento socializador clave y preponderante (cf. Lendon, 1997). No es de extrañar, por tanto, que los dos hermanos Metelos pasaran a convertirse tras el divorcio de su hermanastra en enemigos "oficiales" de Pompeyo.

Los testimonios de la infidelidad de Mucia se encuentran también en fuentes más tardías, así Zonaras $(10,5)$, que sigue a Plutarco prácticamente al pie de la letra y Jerónimo (adv. Jovin., 1316), que cuenta cómo Pompeyo fue el último en enterarse de la infidelidad de su esposa, hecho que al conocerlo le causó una gran tristeza. Dion, por su parte $(37,49,3)$, menciona el divorcio: "Metelo, airado porque había repudiado a su hermana a pesar de tener hijos de ella (...)" (BCG, trad. José Ma Candau Morón), pero no la infidelidad.

¿Qué podemos sacar en conclusión sobre esta cuestión analizando las fuentes del período? El único comentario prácticamente contemporáneo al episodio es el de Cicerón en su carta a Ático de enero de 61. A diferencia de Haley (1985: 51), pensamos que una posible infidelidad no debe descartarse, como motivo principal o al menos como una de las razones, para la disolución del matrimonio. Disentimos también de su afirmación sobre que el testimonio de Cicerón (Fam., 5,2,6) "provides evidence that she [Mucia] was not adulterous" (Haley, ibíd.). Es un dato sintomático que, en la que prácticamente es su única referencia a Mucia en $R R$, Syme alude a ella en su estilo característico como "a woman of flagrant infidelity".

En nuestra opinión, la conclusión de Haley (1985: 52): “(...) there are no solid grounds for condemning Mucia for adultery, political expediency seems, after all, the primary motivation and Mucia's alleged infidelity reveals more about historiography than about her moral behaviour", merece ser matizada.

La razón del divorcio bien pudo ser una suma de ambos factores: por un lado, la infidelidad única o en repetidas ocasiones de Mucia tras el largo período de tiempo pasado por Pompeyo fuera de Roma y, al mismo tiempo y quizá como razón de mayor peso, el hecho palmario de que la infidelidad de su esposa haya proporcionado al ambicioso general el pretexto deseado para realizar nuevas conexiones políticas con otras familias y personajes claves de la política romana, conexiones posibilitadas gracias a una nueva unión matrimonial.

De hecho, tras el divorcio de Mucia, y aunque permaneció tres años sin volverse a casar, Pompeyo intentó un matrimonio sin éxito con la sobrina de Catón

${ }^{5}$ Syme, 1939, 33. Las otras dos citas, en pp. 32 y 228, son referencias al personaje en notas a pie de página. 
(Plut. Pompeyo, 44). Más tarde, su cuarta esposa sería Julia, la hija de Julio César (cf. Humbert, 2000: 76s).

El divorcio de Pompeyo no dinamitó la reputación de Mucia, ni mucho menos, ya que casi veinte años después en 40 y un año después, en 39, la dama adquiere de nuevo un especial protagonismo. Se convierte en un verdadero poder mediador, una arbiter si se nos permite la expresión, que media entre Antonio y, sobre todo, entre su hijo Sexto y Octaviano. Los dos triunviros estaban intentando ganar para su causa a Sexto y a sus efectivos militares y en ese contexto, su madre vuelve a adquirir de nuevo una importante relevancia. Todas las fuentes literarias que tratan el período ponen de relieve este aspecto.

En 40 tras el desenlace de Perusa, si seguimos a Dion (48,15,2 y 48,16,2-3), se produjo una verdadera debacle en el bando antoniano. Fulvia ${ }^{6}$, su mujer, huyó a Grecia y su madre Julia, a la isla de Sicilia en busca de la protección de Sexto. En respuesta a este movimiento, Octaviano "sabedor del poderío militar de aquel [Sex.] y de que negociaba una alianza con Antonio sirviéndose de la madre de éste [Julia] y de los emisarios, temió luchar contra ambos a la vez. Considerando a Sexto más fiable y poderoso que a Antonio, le envió a su madre, Mucia, y se casó con la hermana del suegro de Sexto, Lucio Escribonio Libón [Escribonia]" (D.C., ibíd.) (BCG, trad. Juan Pedro Oliver Segura). Dion se refiere a la que fue la segunda mujer de Octaviano después de divorciarse de Clodia, hija de Fulvia e hijastra de Antonio. En 39, el mismo día que Escribonia daba a luz a Julia, la única hija del futuro princeps, éste se divorció de ella para casarse con Livia, la que a la postre sería su pareja hasta el final de su vida.

Ese mismo año 39, Apiano $(B C 5,39)$ nos narra cómo la plebe de Roma, dentro de la espiral creciente de disturbios sociales de aquellos complejos años, amenazó con quemar a Mucia en su propia casa. Octavio la envió acto seguido para que trabajase su causa ante su hijo Sexto y ese mismo año en la bahía de Nápoles se llegó a un precario acuerdo, una especie de pacto de no agresión, entre Octaviano, Antonio y el propio Sexto.

En concreto Apiano ( $B C 5,72)$, de clara tendencia filoantoniana, habla a las claras de la labor mediadora de Mucia como aglutinadora de voluntades entre su hijo y los triunviros. Ninguna otra fuente, caso de Dion por ejemplo (48,36-38), señala que en la gestación del acuerdo interviniera mujer alguna. En esta cuestión específica sin embargo, nos parece que Apiano se acerca más a lo sucedido que el historiador bitinio.

${ }^{6}$ Está disponible una revisión en positivo del papel histórico de Fulvia en Delia, 1991 y Welch, 1995. Véase también Babcock, 1965 y Fischer, 1999. Y, en general, sobre las esposas de los triunviros véase Christ, 1993. 
El ir y venir diplomático que precedió al tratado de Miseno, cuya famosa escena del banquete de celebración se narra en Plutarco (Ant. 32,3-5), está descrito por Apiano con todo lujo de pormenores $(B C 5,69-73)$. El historiador alejandrino narra cómo Sexto, siguiendo el consejo de sus partidarios y en contra de la opinión de su lugarteniente Menodoro, navegó hasta la isla de Enaria (act. Ischia) con una gran flota, llegando a continuación hasta los alrededores de la bahía de Nápoles $(5,71)$.

Fracasaría una primera entrevista celebrada en Ischia en primavera entre Octavio y Antonio, por un lado, y Sex. Pompeyo y su suegro L. Escribonio Libón (cos. 34, MRR II, 410) por otra. Finalmente, continúa Apiano (5,72), gracias a los esfuerzos y la labor mediadora de Mucia y Julia (un error del historiador por Escribonia), la madre y la esposa de Sexto respectivamente; los dos triunviros y el hijo del conquistador de Oriente volvieron a encontrarse para parlamentar en plena canícula, hacia agosto de 39, en algún lugar indeterminado del arco del golfo formado por las actuales Miseno, Bayas y Puteoli. Aquí vemos otra vez como las fuentes hacen hincapié en la labor de Mucia Tercia como eficaz mediadora en asuntos de alta política.

El acuerdo, conocido para la posteridad como tratado de Miseno, amplió el triunvirato incluyendo a un cuarto socio, Pompeyo que dueño de las islas, recibió también el Peloponeso. Al reconocimiento se sumaba una compensación económica, y futuros consulados para su suegro y para él. Escribonio Libón fue cónsul en 34 y a Sexto se le tenía reservado uno de los de 33. Los proscritos y fugitivos que se habían unido a la causa de éste podían además, si lo deseaban, volver a Italia.

Miseno fue, de largo, el acuerdo de menor entidad de todos los que se plasmaron en la turbia quincena de años que duró aproximadamente el período triunviral. Podríamos calificarlo de pacto meramente coyuntural o de transición. Incluso podríamos hablar de acuerdo fallido, como lo prueba la corta duración del mismo y lo acordado en el siguiente tratado importante, el de Tarento, que supone prácticamente una vuelta al espíritu de Brindisi (Vivas García, 2013: 80s). La corta vigencia de Miseno fue motivada también por el divorcio entre Octaviano y Escribonia ese mismo año. Escribonia era mayor que Octaviano y de carácter difícil y el triunviro puso tierra de por medio a las primeras de cambio. El auténtico desenlace de Miseno fue que, al cabo de poco tiempo, volvieron a sonar tambores de guerra en las costas italianas.

Durante casi una década, hay un vacío sobre Mucia en las fuentes. Pero volvemos a encontrarnos con ella en 31, en relación con las purgas llevadas a cabo por el bando vencedor tras la batalla de Accio. Dion $(51,2,5$ y 56,38,2) nos cuenta como Mucia salvó de la muerte a su hijo M. Emilio Escauro (sen. 43), que había engendrado con el pretor de 56, acusado de ser partidario del derrotado bando antoniano. El texto exacto de Dion dice $(51,2,5)$ : "Marco Escauro (...) era hermano de Sexto por parte de madre y, aunque había sido condenado a muerte [por Octaviano y sus partidarios], más tarde fue perdonado por la intervención de su madre, Mucia" (BCG, trad. Juan Manuel Cortés Copete). Las fuentes nos devuelven de nuevo al primer plano a esta matrona en su faceta más conocida y exitosa, la de mediadora política.

Después de esta breve referencia, el rastro de Mucia desaparece por completo de las fuentes. Ni siquiera conocemos la fecha de su fallecimiento. Simplemente se desvanece en las brumas de la Historia. 


\section{CONCLUSIÓN}

El caso de Mucia Tercia es un ejemplo paradigmático del destacado papel que las mujeres romanas pertenecientes a los grupos de élite alcanzaron durante el último siglo de la República. Las matronae como Mucia consiguieron tener un estatus preponderante en la estructura familiar y social romanas. Durante la República tardía, las mujeres en Roma ocuparon de forma cada vez más preeminente espacios de influencia económica y social, dejando relegados sus roles tradicionales en el ámbito meramente familiar. De hecho los vástagos de la élite, incluidas las féminas ${ }^{7}$, eran criados y alimentados con suma frecuencia por los nutritores. El auténtico papel de la madre romana, y eso se ve de manera palmaria en el caso de Mucia, era el de nexo en la especial relación que la madre mantenía con su hijo adolescente, primero, y adulto, después. En el caso que nos ocupa sucede hasta en tres ocasiones: dos con Sexto Pompeyo en 40 y 39; y ocho años más tarde en 31 mediando de nuevo ante Octaviano para interceder por la vida de su hijo, Marco Escauro.

La matrona romana poseía un rol muy determinado de consejera moral, mentora, guardiana de los valores cívicos en definitiva. Esta función les concernía especialmente si las madres pertenecían a los grupos de mayor poder, como es el caso de Mucia, puesto que estas mujeres eran a menudo independientes en materia económica, lo que les permitía ayudar a sus hijos en la carrera política. De esta circunstancia se derivaba también para estas féminas, un cierto prestigio, honores y un reconocimiento a sus capacidades.

Las señoras romanas eran los custodios más firmes de los valores que definirían posteriormente a un buen ciuis, a un ciudadano ejemplar. Lo paradójico es que ésos eran los valores de una sociedad absolutamente patriarcal, tanto en la organización jurídica y social como en su encuadre mental. Una sociedad que poseía como uno de sus rasgos más definitorios, la búsqueda obsesiva del honos y la reputación, preferentemente a través de la victoria militar. El éxito en los hechos de armas era una de las manifestaciones más perfectas y acabadas de una buena reputación.

Enmarcado en un ethos que podríamos calificar de agonístico, el éxito en la guerra definía al vir perfecto. El aristócrata romano buscaba ser el mejor líder en la batalla y en el gobierno, de la forma más rápida y expeditiva posible. Todo era lícito para conseguir esa meta. Para la mentalidad romana de la élite era plenamente válida la máxima darwiniana del "comer o ser comido". Del mismo modo que la sociedad romana en su conjunto se definía intrínsecamente por la desigualdad entre sus miembros; la aristocracia romana portaba en su ADN la crueldad, la lucha contra el igual, ya que el éxito del aristócrata romano se basaba en la supervivencia de los más aptos.

${ }^{7}$ Sobre la educación característica que recibían las mujeres pertenecientes a la élite social en la antigua Roma, véase la monografía de Hemelrijk, 2004. 
De hecho, esta última frase bien pudiera ser una descripción sintética de lo que supuso para Roma la quincena de años que media entre 44 y 30: la historia de la "supervivencia del más apto". Esa puede ser una atinada y breve descripción de lo que la Historia conoce bajo el nombre de Segundo Triunvirato.

La personalidad de Mucia se ajusta como un guante a lo que acabamos de formular. Es una gran señora política que sirve como mediadora y refrendaria de pactos políticos, el más notorio de ellos el de Miseno en 39. Fue siempre una figura respetada y un referente tanto para su prole como para los enemigos de su familia extensa, caso de Octaviano.

Si hemos conseguido que el lector culto haya aprendido un poco más sobre su apasionante singladura, nos sentimos satisfechos. Ese y no otro era el propósito de estas líneas.

RECIBIDO: octubre 2018; ACEPTADO: noviembre 2018.

\section{REFERENCIAS BIBLIOGRÁFICAS}

BABCOCK, C. L. (1965): "The early career of Fulvia”, The American Journal of Philology 86 (1): 1-32.

Brennan, T. C. (2012): "Perceptions of women's power in the late Republic: Terentia, Fulvia, and the generation of 63 BCE”, en S. Dillon y S. L. JAmes (eds.), A Companion to Women in the Ancient World, Wiley-Blackwell, Oxford, Malden y Chichester, pp. 354-366.

Broughton, T. R. S. (1968): The Magistrates of the Roman Republic vol. II [MRR II], The American Philological Association [reimp.].

Christ, K. (1993): "Die Frauen der Triumvirn", en A. GARA y E. Foraboschi (eds.), Il Triunvirato constituente alla fine della Republica romana, Biblioteca di Athenaeum, Como, pp. 135-153.

Delia, D. (1991): “Fulvia reconsidered”, en S. Pomeroy (ed.), Women's History and Ancient History, University of North Carolina Press, Chapel Hill, pp. 197-217.

$D P R R=$ The Digital Prosopography of the Roman Republic [DPRR], Mucia (28) Tertia, en línea: http://romanrepublic.ac.uk/.

FISCHER, R. A. (1999): Fulvia und Octavia. Die beiden Ehefrauen des Marcus Antonius in den politischen Kämpfen der Umbruchzeit zwischen Republik und Prinzipat, Logos, Berlin.

Fluss, M. (1933): "Mucius 28 (Tertia Mucia)", Real-Encyclopädie der classischen Altertumswissenschaft 16 (1): 449.

GabBA, E. (1970): Appianus. Bellorum Civilium liber quintus, La Nuova Italia, Firenze.

Hadas, M. (1930): Sextus Pompey, Columbia University Press, New York.

Haley, S. P. (1985): "The five wives of Pompey the Great”, Greece \& Rome 32 (1): 49-59.

HARDERS, A.-C. (2015): "Consort or despot? How to deal with a queen at the end of the Roman Republic and the beginning of the Principate", en H. BÖRM (ed.), Antimonarchic Discourse in Antiquity, Franz Steiner Verlag, Stuttgart, pp. 181-214.

HemelRijK, E. A. (2004): Matrona docta: Educated women in the Roman elite from Cornelia to Julia Domna, Routledge, London.

HÖlKeSKAMP, K.-J. (2004): Senatus populusque romanus: die politische Kultur der Republik: Dimensionen und Deutungen, Franz Steiner Verlag, Stuttgart. 
HÖLKeSKAMP, K.-J. (2017): Libera res publica: die politische Kultur des antiken Rom: Positionen und Perspektiven, Franz Steiner Verlag, Stuttgart.

Humbert, M. (1972): Le remariage a Rome. Etude d'histoire juridique et sociale, Giuffre, Milano.

LENDON, J. E. (1997): Empire of honour: the art of government in Roman World, The Clarendon Press, Oxford.

Masi Doria, C. (2014): "Uno scandalo del 52 a.C. e la problemática identità di due donne romane (Nota su Val. Max. 9,1,8)”, en J. HALlebeeK J. et alii (eds.), Inter cives necnon peregrinos: Essays in honour of Boudewijn Sirks, V\&R Unipress, Göttingen, pp. 493-500.

$M R R=$ BROUGHTON, 1968.

Momigliano, A. (1940): "Review of The Roman Revolution”, Journal of Roman Studies 30: 75-80. $R R=$ SYME, 1939.

Seager, R. (2002²): Pompey the Great. A political biography, Blackwell, Oxford.

Stegmann, H. (2000): “Mucia Tertia”, Der Neue Pauly 8, Metzler, Stuttgart-Weimar, pp. 423-424.

Syme, R. (1939): The Roman Revolution, The Clarendon Press, Oxford.

Vivas García, G. A. (2013): Octavia contra Cleopatra. El papel de la mujer en la propaganda política del Triunvirato (44-30 a. C.), Liceus, Madrid.

Welch, K. (1995): "Antony, Fulvia and the Ghost of Clodius in 47 B.C.”, Greece \& Rome 42 (2): 182-201.

Welch, K. (2012): Magnus Pius: Sextus Pompeius and the transformation of the Roman republic, The Classical Press of Wales, Swansea.

ZMesKal, K. (2009): Adfinitas. Die Verwandtschaften der senatorischen Fuhrungsschicht der romischen Republik von 218-31 v. Chr., K. Stutz, Passau. 